This is an Open Access article, distributed under the terms of the Creative Commons Attribution licence (http://creativecommons.org/licenses/by/4.o/), which permits unrestricted re-use, distribution, and reproduction in any medium, provided the original work is properly cited.

doi:10.1017/So144686X17000034

\title{
Social support networks of older migrants in England and Wales: the role of collectivist culture
}

\author{
VANESSA BURHOLT*, CHRISTINE DOBBS* and \\ CHRISTINA VICTOR $\dagger$
}

\begin{abstract}
This article tests the fit of a social support network typology developed for collectivist cultures to six migrant populations living in England and Wales. We examine the predictive utility of the typology to identify networks most vulnerable to poor quality of life and loneliness. Variables representing network size, and the proportion of the network classified by gender, age, kin and proximity, were used in confirmatory and exploratory latent profile analysis to fit models to the data $(\mathrm{N}=815$; Black African, Black Caribbean, Indian, Pakistani, Bangladeshi and Chinese). Multinomial logistic regression examined associations between demographic variables and network types. Linear regression examined associations between network types and wellbeing outcomes. A four-profile model was selected. Multigenerational Household: Younger Family networks were most robust with lowest levels of loneliness and greatest quality of life. Restricted Non-kin networks were least robust. Multigenerational Household: Younger Family networks were most prevalent for all but the Black Caribbean migrants. The typology is able to differentiate between networks with multigenerational households and can help identify vulnerable networks. There are implications for forecasting formal services and variation in networks between cultures. The use of a culturally appropriate typology could impact on the credibility of gerontological research.
\end{abstract}

KEY WORDS - ethnicity, familism, communalism, loneliness, quality of life.

* Centre for Innovative Ageing, College of Human and Health Sciences, Swansea University, UK.

$\dagger$ College of Health and Life Sciences, Brunel University London, Uxbridge, UK. 


\section{Vanessa Burholt et al.}

\section{Introduction}

Models, measures and typologies of social support networks have predominantly been developed in individualistic rather than collectivist cultures. An individualistic culture is defined as one in which the members value independence, and the cultural norm is for nuclear living arrangements (i.e. a single person, couple, or couple and young children only). On the other hand, 'collectivist cultures' value interdependence and are oriented towards cohesion, commitment and obligation. In collectivist cultures, social units with common goals are central. Consequently, collectivist value systems are strongly related to communalism, familism and filial piety (Schwartz et al. 2010). 'Communalism' emphasises social bonds to kin and non-kin, and prioritises social relationships over individual achievement. 'Familism' prioritises the family (as a social unit) over the individual needs and 'filial piety' emphasises respect for older family members and obligations towards meeting parents' needs (Schwartz et al. 2010; Triandis 1989).

Social support networks are the configuration of relationships that have the potential to provide emotional, instrumental and social support to an older person. While these instruments have utility in exploring the potentiality of support in individualistic cultures, in collectivist cultures where there is often a preponderance of multigenerational households this approach leads to skewed distributions of support resources and an under-estimation of the proportion of older people who may require additional help (Burholt and Dobbs 2014).

In this article we test the fit of a four-class social support network typology that has been developed within collectivist cultures (Burholt and Dobbs 2014) to a unique sample comprising a diverse population of six migrant groups living in the United Kingdom (UK) (Black African, Black Caribbean, Indian, Pakistani, Bangladeshi and Chinese). Our main research question is: Does the structure of the network typology fit a broad range of collectivist cultures? We supplement this primary question with two secondary questions: Are differences observed in the distribution of network types between ethnic groups? Does the network typology have predictive utility?

This article is innovative and focuses on an understudied field, that is, older migrants in Europe. We provide a deeper insight into the support networks of older migrants: a population that is growing currently in many European countries. We describe the characteristics of the networks and examine the ability of the network typology to predict outcomes that are unrelated to the variables used in the clustering, but that are theoretically 
related to the profiles by considering if network type is associated with loneliness and quality of life (Henry, Tolan and Gorman-Smith 2005).

\section{Culturally appropriate measures and models of social support}

It is often assumed uncritically that measures and/or typologies that are developed in individualistic cultures such as North America, Northern and Western Europe, and Australia can be applied to other socio-cultural contexts (Lubben and Gironda 2003). However, this assumption fails to take into account differences between individualistic and collectivist cultures such as those found in most of Latin America, Asia and Africa, in terms of normative family living arrangements and the primacy of kin or non-kin relationships (Hofstede 2001; for exceptions, see Dubova et al. 2010; in China: Cheng et al. 2009).

Social support models or measurements are often generated by considering the proximity and frequency of contact with family members as, for example, by the Wenger Support Network Typology (Wenger 1991), the Lubben Support Network Scale (Lubben and Gironda 2004) and the Litwin Support Network Types (Litwin 1997). When quantifying social support, proximal living arrangements in collectivist cultures result in frequent contact between generations which are often operationalised as higher levels of resources and are skewed towards robust networks (Bangladesh: Burholt et al. 200o; China: Wenger and Lui 1999). However, frequent contact may not necessarily translate into support resources (Willis 2008) and most network typologies are unable to make distinctions between different configurations of social support within mutigenerational households, or within collectivist cultures in general. In individualist cultures such as the UK, Northern Europe, Australasia and North America, it is essential that we use adequate tools that are fit for purpose and recognise cultural diversity (Burholt et al. 2007; Whitfield et al. 2008).

A support network typology developed for older people within collectivist cultures identified four types of support networks among older South Asians (Indian Gujaratis, Indian Punjabis and Bangladeshis living in the UK and in South Asia) (Burholt and Dobbs 2014). The configurations of relationships were named 'Multigenerational Household: Older Integrated', 'Multigenerational Household: Younger Family', 'Family and Friends Integrated' and 'Restricted Non-kin' networks, and were differentiated on the structure of the networks, community integration, and the quantity of support provided and received. The new typology distinguished between two networks associated with multigenerational households and performed better than the Wenger Network Typology (Wenger 1991). Using the 
Wenger Network Typology, a small minority of South Asian elders (4.2\%) were identified as embedded in private restricted supports networks compared with nearly a fifth $(18.2 \%)$ classified as having Restricted Non-kin networks by the new typology.

Collectivism is presumed to apply to cultures in regions other than South Asia, including Latin America, South East Asia, Africa and the Middle East (Triandis 1995), but this proposition needs to be substantiated with research (Schwartz et al. 2010). Our first hypothesis is that the structure of the network typology developed with South Asian elders will fit a sample comprising six migrant groups (Black Caribbean, Black African, Chinese, Indian, Pakistani and Bangladeshi) with collectivist cultures in the UK.

\section{Differentiation between collectivist cultures}

In 2011 , the UK Census data indicated that the percentages of the population aged $6_{5}$ or over were 2.4 per cent of Black Africans, 13.9 per cent of Black Caribbeans, 8.1 per cent of Indians, 4.4 per cent of Pakistanis, 3.7 per cent of Bangladeshis and 5 .o per cent of Chinese. Cultures in the countries of origin of the six migrant groups in this study may be broadly described as having a collectivist orientation, whereas the White British population is described as individualistic (Willis 2012). However, cultures do not align to a single dimension from individualism to collectivism (Knight and Sayegh 2009) as there are slightly different manifestations of values concerning communalism, familism and/or filial piety (Schwarz et al. 2010). Moreover, the degree to which the cultures in the country of origin are adhered to in the UK may be influenced by acculturation and cultural identity.

In the United States of America (USA), research suggests that African Americans, Caribbean Blacks and African immigrants have communalist value orientations. In this respect, 'families' comprise both kin and nonkin relations (Wallace and Constantine 2005). Thus, the configuration of relationships within networks for migrants from communalist cultures is likely to differ from other collectivist cultures where non-kin relationships have lower priority. We expect the communalist value orientation to be replicated in the UK, and our second hypothesis posits that Black Caribbean and Black African participants will have a greater proportion of friends-based networks than other collectivist cultures.

Whereas collectivist cultures originating from Africa and the Caribbean may be described as communalist, those stemming from South and SouthEast Asia are described as familistic and emphasise filial piety (Cheng, Fung and Chan 2007). We would expect these cultural identities to be 
reflected in familistic types of social support networks in the UK and hypothesise that Chinese, Bangladeshi, Pakistani and Indian migrants will have a greater proportion of family-based networks than Black Caribbean and Black African migrants (Hypothesis 3).

While Chinese culture is often described as familistic with strong filial piety (Cheng, Fung and Chan 2007) and Black Caribbean culture as communal (Wallace and Constantine 2005), there are specific circumstances impacting on these populations in the UK that lead us to believe that the distributions of networks may not entirely reflect these collectivist cultures in the family's country of origin. Research has shown that Chinese and Black Caribbean migrants had a weaker cultural identity with the family's country of origin and ethnic group than did other migrant groups (Burholt, Dobbs and Victor 2016). We expect the weaker cultural identities of Chinese and Black Caribbean migrants in the UK to manifest in more fragile social ties or less collectivistic forms of social support networks. Our fourth hypothesis is that Chinese and Black Caribbean migrants will have a greater proportion of restricted or private networks than the other migrant groups.

\section{Outcomes of social support in cultural contexts}

Social comparison theory (Festinger 1954) posits that social and personal worth are determined by perceptions of how others fare in relation to one's own position. Culturally located comparisons of social support may be conceptualised quite differently in individualistic and collectivist cultures. While in general, meagre social support is related to poor quality of life and worse levels of anxiety and loneliness (Golden, Conroy and Lawlor 2009; Lubben and Gironda 2003), the configuration of support also has an impact on wellbeing outcomes (Knight and Sayegh 2009).

In the UK, older people embedded in networks that provide family contact only, or very little social contact with others, experience greater levels of loneliness than those embedded in networks that provide high levels of interaction with friends and family (Wenger et al. 1996). This would suggest that in the UK the norm is to have a mixture of family and friends within a network, and that the consequence of social comparison, whereby one's situation is found to deviate from the norm, has negative outcomes. Conversely, in general a collectivistic orientation emphasises family responsibility (Knight and Sayegh 2009). Despite anticipated differences between the six migrant groups in the distribution of network types, we would still expect poor wellbeing outcomes for individuals when expectations concerning family responsibilities are not fulfilled (Triandis et al. 1988; van de Vijver and Arends-Tóth 2009). We hypothesise that migrants 


\section{$145^{8}$ Vanessa Burholt et al.}

with family-oriented networks will experience (Hypothesis 5 ) lower levels of loneliness and (Hypothesis 6 ) better quality of life than those with diverse or restricted networks.

\section{Methods}

The data used in this article arise from the study 'Inter, Intra-generational and Transnational Caring in Minority Communities in England and Wales'. This project examined the prevalence of informal care amongst six major minority ethnic groups. The study population comprised adults aged 40 years and over from six ethnic groups: Black Caribbean, Black African, Indian, Pakistani, Bangladeshi and Chinese people living in England and Wales. The target sample size was 1,200 (stratified as 100 persons per ethnic and generational group: 6oo aged $40-64$ years and 6 oo aged $65^{+}$ years). A face-to-face survey was conducted with $\mathrm{N}=1,206$ people. Indepth qualitative interviews were conducted with $\mathrm{N}=60$ participants (five from each of the six ethnic and two generational groups). This article is based on a sub-sample of $\mathrm{N}=815$ older people aged 55 years or more. The age threshold of the sub-sample was based on previous studies of older migrants in the UK, the shorter life expectancy of some of the migrant groups and the relative youth of the immigrant population in the UK (Burholt 2004a, 2004b).

\section{Procedure}

Sampling points were identified for each ethnic group in England and Wales using the Postcode Address File (PAF) that divides the UK into districts comprising around 30,000 postcodes (Royal Mail 2009). Data files were constructed for populations of each ethnic group organised at the PAF locality level drawing on information from the UK Census 2001. The PAF localities were ordered according to the population size of each ethnic group, separately for each ethnic group in England and each ethnic group in Wales (12 lists in total) and systematic random sampling was used to select sampling points on each list.

The interview schedule was compiled in English and included items from a project conducted in South Asia and Birmingham (Burholt and Dobbs 2014) and from the Survey of Household Carers 2009-2010 (National Health Service Information Centre 2010). For the majority of survey questions, conceptual and functional equivalence was straightforward: these items were translated directly from English to Punjabi, Gujarati, Hindi, Mandarin Chinese, Bengali, Somali, Yoruba or Urdu during the course of 
the interview. This method of translation is standard practice for the data collection agency (e.g. Grant and Bowling 2011). Nineteen questions were translated into the eight languages using front-back translation methods (Koller et al. 2007).

Interviews were conducted by a market research group (Ethnic Focus) between October 2011 and April 2012 in the respondent's native language or in English and, wherever possible, in the respondents' own homes. Potential participants approached by Ethnic Focus that did not identify with one of the six ethnic groups were not eligible for inclusion in the study, and were not interviewed.

\section{Sample}

Overall, the response rate was 40 per cent (Black African: $4^{1}$ per cent; Black Caribbean: $4^{1}$ per cent; Indian: $4^{8}$ per cent; Pakistani: 44 per cent; Bangladeshi: $4^{1}$ per cent; Chinese: 27 per cent). The net final size was $\mathrm{N}=1$,206. The sub-sample of older people aged 55 years or more comprised $\mathrm{N}=815$ people (Black African: $\mathrm{N}=143$; Black Caribbean: $\mathrm{N}=162$; Indian: $\mathrm{N}=141$; Pakistani: $\mathrm{N}=141$; Bangladeshi: $\mathrm{N}=134$; Chinese: $\mathrm{N}=94$ ).

Black Caribbean participants were on average the oldest participants $($ mean $=68.08$, standard deviation $(\mathrm{SD})=6.82), F(5,809)=2.27, p<0.05$, and were more likely than other participants to be never married, divorced or separated. In all other ethnic groups, a majority of participants were married, and between one-quarter and one-third were widowed, $\chi^{2}\left(1_{5}\right.$, $\mathrm{N}=815)=90.98, p<0.001$. There were no differences between ethnic groups in gender of the participants, with the male to female ratio

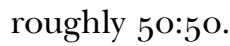

\section{Measures}

Network membership. An older person's network (aged 16 years or more) comprised household members, up to five friends (generated in response to the question 'who are your closest friends that you see most frequently?') and people that were named in responses to questions that asked about functional sources of help (when ill, buying food, cooking and household chores), informational sources of help (financial advice) and emotional support (when unhappy and discussing a personal problem). Six new variables were created to represent (a) network size, and the proportion of the network that were (b) male; in each of three age groups $(<45$ years (redundant parameter), (c) $45^{-6} 4$ years and (d) $65^{+}$years); (e) kin; and (f) proximity (living in the participant's household or not) (Burholt and Dobbs 2014). 
Loneliness. Loneliness was measured using the six-item De Jong Gierveld scale. The score is the sum of all items, where higher scores represent greater levels of loneliness. The six-item scale has a reported alpha coefficient of reliability ranging from 0.70 to 0.76 (De Jong Gierveld and Van Tilburg 2006) and in the present study was 0.65 .

Quality of life. Quality of life was replicated from the Survey of Household Carers 2009-2010 (National Health Service Information Centre 2010) and assessed by a single item using a five-point Likert-type scale. Participants were asked "If we were to define "quality of life" as how you feel overall about your life, including your standard of living, your surroundings, friendships and how you feel day-to-day, how would you rate your quality of life?' Quality of life ratings ranged from very good (1) to very bad (5) (see also Walthery et al. 2015).

Co-variates. Demographic covariates used in the analysis were age, gender (male/female), marital status (married; never married; widowed; divorced or separated), ethnicity and self-assessed health. Self assessed health ranged from good (1), fair (2) to poor (3).

\section{Analytical procedure}

Using Mplus version 5 we undertook confirmatory and exploratory latent profile analysis with six indicators to test the fit of the support network model to the data (Figure 1). In the first step, the hypothesised confirmatory model (Model 1 ) was run. The mean network size was considerably smaller $($ mean $=3.89, \mathrm{SD}=1.89)$ than the four-cluster development model sample (mean $\left.=5 \cdot 5^{8}, \mathrm{SD}=2.25\right)$, so values were set relative to the distribution of means in the development sample (Table 1). Thus, the start value for network size for the Multigenerational Household: Older Integrated and the Family and Friends Integrated networks was calculated as the mean network size plus one (4.89); the start value for network size for Multigenerational Household: Younger Family networks was set at the mean; and the start value for Restricted Non-kin network was the mean minus one (2.89). In the confirmatory model, mean values for network size were left free to vary, but all other start values were constrained and set at mean values identified for the four-cluster development model (Table 1).

In the second step, the fit of the confirmatory model was compared to three exploratory models with three (Model 2), four (Model 3 ) and five profiles (Model 4 ). In these models, no start values were stipulated. In the third step, the confirmatory model was compared to a four-profile 


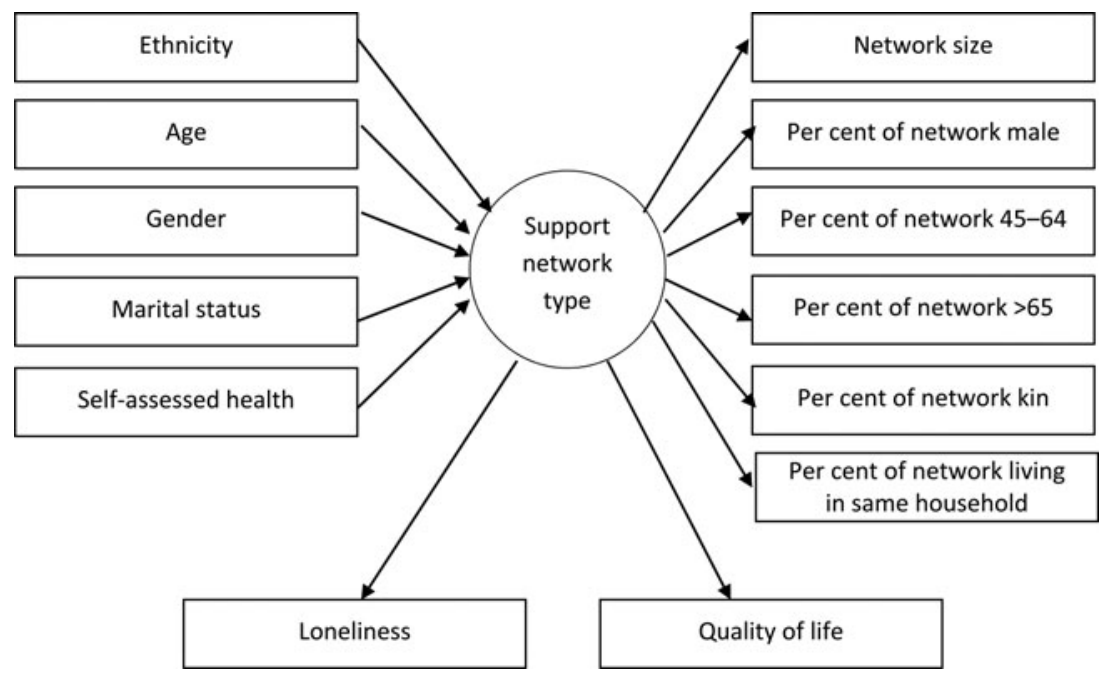

Figure 1. Hypothesised latent profile model of support network types being tested (on the basis of Burholt and Dobbs 2014) with socio-demographic co-variates and loneliness and quality of life as wellbeing distal outcomes.

model (Model 5 ). In this model start values were stipulated as in Table 1 with the exception of network size which was predetermined as above. The start values were free to vary for three of the four profiles (Multigenerational Household: Older Integrated; Multigenerational Household: Younger Family and Family and Friends networks) but were constrained for the Restricted Non-kin network.

Models were compared using Akaike's Information Criterion (AIC; Akaike 1987) and the sample size-adjusted Bayesian Information Criterion (Sclove 1987) in which smaller values represent better fit. We assessed the relative adequacy of the fit of the model using the Vuong-LoMendell-Rubin (Vuong 1989) adjusted likelihood ratio test and the bootstrapped parametric likelihood ratio test (McLachlan and Peel 2000). Both tests compare the model with $K$ classes to a model with $K-1$ classes. Entropy captured how distinguishable classes are from each other with values closer to 1 indicating clear delineation of classes (Celeux and Soromenho 1996). Theoretical reasoning was also used to judge the adequacy of the models (Nylund 2007).

The selected model was used to classify participants based on the most probable class membership. Analysis of variance of means was used to compare network profiles. Class membership was used as the dependent variable in a multinomial regression to establish how demographic covariates including ethnicity were related to network type. 
T A B LE 1. Defining characteristics of network members in the four-cluster development model of network types (Burholt and Dobbs 20I4) used as start values in Models I and $5^{I}$

\begin{tabular}{|c|c|c|c|c|c|c|c|}
\hline \multirow[b]{2}{*}{ Network type } & \multirow{2}{*}{$\begin{array}{l}\text { Mean } \\
\text { network } \\
\text { size }\end{array}$} & \multirow[b]{2}{*}{ Male $^{2}$} & \multicolumn{3}{|c|}{ Age } & \multirow[b]{2}{*}{$\mathrm{Kin}^{2}$} & \multirow{2}{*}{$\begin{array}{l}\text { Living in } \\
\text { same } \\
\text { household }\end{array}$} \\
\hline & & & $\begin{array}{l}<45 \\
\text { years }\end{array}$ & $\begin{array}{l}45^{-64} \\
\text { years }^{2}\end{array}$ & $\begin{array}{l}\geqslant 65 \\
\text { years }^{2}\end{array}$ & & \\
\hline $\begin{array}{l}\text { Multigenerational } \\
\text { Household: Older } \\
\text { Integrated }\end{array}$ & 5.82 & $5^{1.93}$ & 39.97 & 12.84 & $47 \cdot 59$ & $69 \cdot 39$ & $59 \cdot 64$ \\
\hline $\begin{array}{l}\text { Multigenerational } \\
\text { Household: } \\
\text { Younger Family }\end{array}$ & $5 \cdot 57$ & $47 \cdot 79$ & 70.54 & 20.69 & 8.83 & 87.79 & 81.84 \\
\hline $\begin{array}{l}\text { Family and Friends } \\
\text { Integrated }\end{array}$ & 5.85 & $5^{8.70}$ & 25.89 & $5^{8.80}$ & $15 \cdot 31$ & $4^{8.72}$ & 37.67 \\
\hline Restricted Non-kin & 4.81 & $3^{8.57}$ & 11.81 & 9.83 & 69.95 & 26.44 & 13.54 \\
\hline All & $5 \cdot 5^{8}$ & $5^{0.20}$ & 36.27 & 26.73 & 32.49 & 61.03 & $5^{1.4^{2}}$ \\
\hline
\end{tabular}

Notes: 1. Except for the start values for $\mathrm{M}_{1}$ and $\mathrm{M}_{5}$, which are reported in the text. 2. Mean values for the proportion of network with this characteristic.

Two linear regressions were run with loneliness and quality of life as dependent variables. In the first step, age, gender, marital status, ethnic group and health were entered into the respective models. In a second step, variables representing the probability of classification to network profiles were entered into the model to establish the extent to which network type contributed to the variance in the outcome variables. The statistical significance for all tests was set at $p<0.05$.

\section{Results}

\section{Latent profile analysis}

The exploratory five-profile model (Model 4 ) had the best fit on all model fit indicators (Table 2). However, only a very small number of respondents were assigned to the profile corresponding to Restricted Non-kin networks $(\mathrm{N}=19)$. This comprised overwhelmingly $(99 \%)$ of older network members $\left(65^{+}\right.$years $)$who lived in the same household. Taking into account social comparison theory, one would expect that older people with smaller networks than normative in the cultures included in the study (i.e. fewer than four members) would consider themselves to be isolated in comparison to others. We concluded that in Model 4 the restricted network type was unrealistically strictly defined by the model, because it captures only the most isolated 5 per cent of our study population. Because of the 
T А В L E 2. Fit statistics for latent profile models

\begin{tabular}{|c|c|c|c|c|c|c|}
\hline Model & Description & AIC & $\mathrm{BIC}$ & Entropy & VLMR & Parametric bootstrapped LR test \\
\hline 1 & Confirmatory: four profiles & $4^{1,292.55^{6}}$ & $4^{1,335 \cdot 329}$ & 0.76 & $p<0.001$ & $p<0.001$ \\
\hline 2 & Exploratory: three profiles & $4^{1,501.15^{2}}$ & $41,540.869$ & $0.8_{5}$ & $p<0.001$ & $p<0.001$ \\
\hline 3 & Exploratory: four profiles & $41,197.121$ & $41,247.531$ & 0.87 & $p<0.001$ & $p<0.001$ \\
\hline 4 & Exploratory: five profiles & $40,905 \cdot 978$ & $40,967.081$ & 0.90 & $p<0.01$ & $p<0.001$ \\
\hline 5 & Four-profile model with one class constrained & $41,273.090$ & $41,404 \cdot 779$ & 0.81 & $p=0.05$ & $p<0.001$ \\
\hline
\end{tabular}

Notes: AIC: Akaike's Information Criterion. BIC: sample size-adjusted Bayesian Information Criterion. VLMR: Vuong-Lo-Mendell-Rubin adjusted likelihood ratio (LR) test. 


\section{Vanessa Burholt et al.}

limitations associated with classification to Restricted Non-kin networks, we rejected Model 4 (and subsequently Model 3 on the same grounds).

Model 5 yielded an acceptable model fit: the fit was slightly inferior to the exploratory models, but had slightly superior AIC and entropy values compared to our confirmatory model (Table 2). Furthermore, the characteristics of the profiles closely resembled those in the four-cluster development typology (Table 3 ). Model 5 was selected based on model fit, profile characteristics and theoretical reasoning.

\section{Characteristics of network types}

Table 3 provides data on characteristics of the profiles. Overall, the profiles were similar to the development model and the network names were retained to reflect the characteristics of the selected model. There was one exception: the former Family and Friends Integrated network was renamed Middle-aged Friends because the networks had proportionally fewer kin $(28.1 \%$ versus $48.7 \%)$ and fewer members living in the same household $(22.2 \%$ versus $37.7 \%)$ in the confirmatory model than in the development model (Burholt and Dobbs 2014). The networks were described as follows.

Multigenerational Household: Younger Family networks were the largest, comprising on average nearly five members, and accounted for nearly half of all networks $(49.1 \%)$. Networks comprised overwhelmingly of kin $(73.3 \%)$ with only around one-quarter non-kin members $(26.7 \%)$. These networks had the greatest proportion of members living in the same household: around two-thirds $(67.3 \%)$ of network members co-resided. A vast majority of network members were younger than $6_{5}(82.4 \%)$ with a majority under 45 years $(43.4 \%)$. This network typically comprised three or more generations often living in the same house. Of all the network types, this profile was the most family-focused.

Nineteen per cent of the sample was assigned to Multigenerational Household: Older Integrated networks. These networks were typically smaller than the Multigenerational Household: Younger Family networks (three versus five members). Networks comprised around two-thirds kin $(66.1 \%)$ to one-third non-kin $(33.9 \%)$, suggesting that relationships were focused both within and outside the household. Although the networks included younger and older members, on average more than two-thirds $(68.3 \%)$ of network members were over 65 years old. Over half of the network members lived in the same household $(57.8 \%)$. Therefore, the size of the network and the proportion of the network that was over $6_{5}$ years and non-kin served to differentiate it from the other multigenerational network type. 
T А в L E 3. Defining characteristics of networks in the final model (Model 5): estimated means and observed means based on most probable class membership

\begin{tabular}{|c|c|c|c|c|c|c|c|c|c|c|c|c|c|c|c|}
\hline \multirow{3}{*}{$\begin{array}{l}\text { Network } \\
\text { type }\end{array}$} & \multirow[b]{3}{*}{$\mathrm{N}$} & & & & & \multicolumn{6}{|c|}{ Age } & \multirow{2}{*}{\multicolumn{2}{|c|}{$\operatorname{Kin}^{1}$}} & \multirow{2}{*}{\multicolumn{2}{|c|}{$\begin{array}{l}\text { Living in same } \\
\text { household }^{1}\end{array}$}} \\
\hline & & \multicolumn{2}{|c|}{ Network size } & \multicolumn{2}{|c|}{ Male $^{1}$} & \multicolumn{2}{|c|}{$<45$ years $^{1}$} & \multicolumn{2}{|c|}{$45^{-64}$ years $^{1}$} & \multicolumn{2}{|c|}{$\geqslant 6_{5}$ years $^{1}$} & & & & \\
\hline & & Est & $\mathrm{Obs}^{3}$ & Est & $\mathrm{Obs}^{3}$ & Est $^{2}$ & $\mathrm{Obs}^{3}$ & Est & $\mathrm{Obs}^{3}$ & Est & $\mathrm{Obs}^{3}$ & Est & $\mathrm{Obs}^{3}$ & Est & $\mathrm{Obs}^{3}$ \\
\hline MYF & 400 & $\begin{array}{c}3.17 \\
(0.16)\end{array}$ & $\begin{array}{c}4.81 \\
(2.01)\end{array}$ & $\begin{array}{l}49.06 \\
(3.65)\end{array}$ & $\begin{array}{c}4^{6.83} \\
(20.61)\end{array}$ & 8.88 & $\begin{array}{c}\mathbf{4 3 . 3 7} \\
(22.09)\end{array}$ & $\begin{array}{c}78.83 \\
(3.54)\end{array}$ & $\begin{array}{c}39.01 \\
(20.66)\end{array}$ & $\begin{array}{l}12.29 \\
(2.19)\end{array}$ & $\begin{array}{c}I 7.63 \\
(16.70)\end{array}$ & $\begin{array}{l}29.09 \\
(3.39)\end{array}$ & $\begin{array}{c}73 \cdot 30 \\
(16.68)\end{array}$ & $\begin{array}{l}22.3^{2} \\
\left(2.8_{4}\right)\end{array}$ & $\begin{array}{c}\mathbf{6 7} \cdot \mathbf{3 2}^{2} \\
(18.48)\end{array}$ \\
\hline MOI & $15^{6}$ & $\begin{array}{c}3.14 \\
(0.26)\end{array}$ & $\begin{array}{c}3.12 \\
(1.40)\end{array}$ & $\begin{array}{l}48.00 \\
\left(2.5^{1}\right)\end{array}$ & $\begin{array}{c}47.17 \\
(28.09)\end{array}$ & $25 \cdot 44$ & $\begin{array}{c}27 \cdot 44 \\
\left(23 \cdot 3^{8}\right)\end{array}$ & $\begin{array}{c}7.94 \\
(4.64)\end{array}$ & $\begin{array}{c}4 \cdot 3^{I} \\
(9.62)\end{array}$ & $\begin{array}{c}66.62 \\
(4.73)\end{array}$ & $\begin{array}{c}68.25 \\
(23.11)\end{array}$ & $\begin{array}{l}64.99 \\
(4.67)\end{array}$ & $\begin{array}{c}66.06 \\
(18.94)\end{array}$ & $\begin{array}{c}5^{6.35} \\
(5 \cdot 54)\end{array}$ & $\begin{array}{c}57.80 \\
\left(20.8_{5}\right)\end{array}$ \\
\hline MAF & 116 & $\begin{array}{c}4.85 \\
(0.12)\end{array}$ & $\begin{array}{c}3.27 \\
(1.30)\end{array}$ & $\begin{array}{l}4^{6.40} \\
(1.09)\end{array}$ & $\begin{array}{c}47 \cdot 9^{2} \\
(33 \cdot 45)\end{array}$ & 43.68 & $\begin{array}{c}9.43 \\
(16.02)\end{array}$ & $\begin{array}{l}3^{8} \cdot 34 \\
\left(1.5^{6}\right)\end{array}$ & $\begin{array}{c}79 \cdot 91 \\
\left(19 \cdot 5^{8)}\right.\end{array}$ & $\begin{array}{l}17 \cdot 4^{8} \\
(1.98)\end{array}$ & $\begin{array}{c}I 0.66 \\
\left(15.5^{\mathrm{O}}\right)\end{array}$ & $\begin{array}{l}72.91 \\
(1.74)\end{array}$ & $\begin{array}{c}28.07 \\
(19.72)\end{array}$ & $\begin{array}{l}67.07 \\
(2.25)\end{array}$ & $\begin{array}{c}22.17 \\
(20.13)\end{array}$ \\
\hline RNK & 143 & $\begin{array}{c}2.63 \\
(0.20)\end{array}$ & $\begin{array}{c}2.67 \\
(1.48)\end{array}$ & $\begin{array}{l}38.57 \\
(0.00)\end{array}$ & $\begin{array}{c}43.85 \\
(37.07)\end{array}$ & 20.22 & $\begin{array}{c}18.72 \\
(31.43)\end{array}$ & $\begin{array}{c}9.83 \\
(\text { o.oo })\end{array}$ & $\begin{array}{c}I 2.2 I \\
(16.72)\end{array}$ & $\begin{array}{l}69.95 \\
(0.00)\end{array}$ & $\begin{array}{c}\mathbf{6 9 . 0 7} \\
(31.61)\end{array}$ & $\begin{array}{l}26.44 \\
(0.00)\end{array}$ & $\begin{array}{c}22.5^{2} \\
\left(20.4^{6}\right)\end{array}$ & $\begin{array}{l}13.54 \\
(0.00)\end{array}$ & $\begin{array}{c}12.18 \\
(14.92)\end{array}$ \\
\hline All & 815 & & $\begin{array}{l}3.89 \\
(1.95)\end{array}$ & & $\begin{array}{c}46.53 \\
(27.53)\end{array}$ & & $\begin{array}{l}31.16 \\
(26.87)\end{array}$ & & $\begin{array}{c}33.49 \\
(29.91)\end{array}$ & & $\begin{array}{c}35.35 \\
(33.09)\end{array}$ & & $\begin{array}{c}5^{6.57} \\
\left(28.4^{1}\right)\end{array}$ & & $\begin{array}{c}49 \cdot 40 \\
(29 \cdot 44)\end{array}$ \\
\hline
\end{tabular}

Notes: MYF: Multigenerational Household: Younger Family. MOI: Multigenerational Household: Older Integrated. MAF: Middle-aged Friends. RNK: Restricted Non-kin. Est: estimated (mean with standard error in parentheses). Obs: observed after classification into most probable class membership (mean with standard deviation in parentheses). 1. Proportion of network with this characteristic.2. Redundant parameter: estimated mean based on values for $45^{-64}$ years and $\geqslant 65$ years. 3 . Analysis of variance demonstrated significant differences between network types for network size $F(3$, 811$)=$ $76.84, p<0.001 ;<45$ years $F(3,811)=83.50, p<0.001 ; 45^{-64}$ years $F(3,181)=462.87, p<0.001 ; \geqslant 65$ years $F(3,811)=392.43, p<0.001 ;$ kin $F(3$, $811)=385.49, p<0.001$; living in same household $F(3,811)=406.31, p<0.001$. Post hoc group comparisons - Tukey test: numbers that appear in bold (e.g. 4.81) constitute sub-sets with the highest values; numbers that appear italic $(e . g .4 \cdot 3 I)$ constitute sub-sets with the lowest values. 
Middle-aged Friends networks were relatively uncommon, with only 14 per cent of participants classified in this group. The size of networks was average (around three people), but more than three-quarters $(77.8 \%)$ of network members lived in a different household. The key difference between this network type and the multigenerational household networks was the proportion of non-kin members: networks comprised mainly friends $(71.9 \%)$ aged between 45 and 64 years $(79.9 \%)$. The characteristics suggest that older people with this type of network had a community-facing lifestyle.

Fewer than one-fifth $(17.6 \%)$ of the sample were assigned to Restricted Non-kin networks. These networks were similar to Middle-aged Friends networks but were smaller, containing on average fewer than three members, and older, with more than two-thirds $(69.1 \%)$ over $6_{5}$ years old. These small networks had a large proportion of non-kin members $(77.5 \%)$. These networks may be disconnected from local communities or families.

While the network typology differs slightly from the development model fitted to a sample of South Asian elders, our first hypothesis is supported as the structure of the network typology generally fits the sample comprising six migrant groups with collectivist cultures in the UK. We can broadly determine a hierarchy concerning the dominance of family and friends within the networks. Multigenerational Household: Younger Family networks are dominated by kin relationships and are primarily household-focused, whereas Middle-aged Friends are dominated by non-kin relationship and are community-facing. The Multigenerational Household: Older Integrated network falls somewhere between the two: while kin relationships are important, so too are non-kin in these smaller configurations of social ties. The Restricted Non-kin network is the smallest network and an older person with this type of network has few people to draw upon for support or social interaction.

\section{Differences in network types between ethnic groups}

Figure 2 shows the distribution of network types across the six ethnic groups. Across all groups the Multigenerational Household: Younger Family network was most prevalent for all but the Black Caribbean group. Significant differences are observed between the groups, $\chi^{2}\left(8_{15}, \mathrm{~N}=15\right)$ $=80.89, p<0.001$ : older Black Caribbean people had the greatest proportion of Middle-aged Friends and Restricted Non-kin networks and, alongside Chinese elders, had the fewest Multigenerational Household: Younger Family networks. On the other hand, Pakistani and Bangladeshi elders had proportionally more Multigenerational Household: Younger 


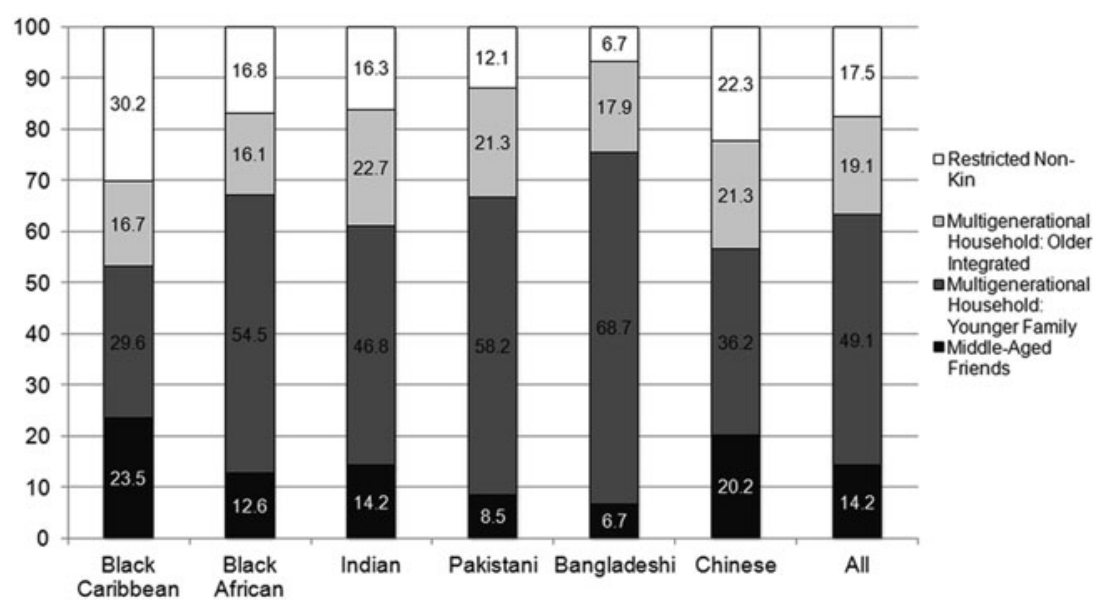

Figure 2. Distribution of network type across six ethnic groups of migrants (\%).

Family networks than the other ethnic groups. Bangladeshi elders had fewer Middle-aged Friends networks than other groups.

The results of multinomial logistic regression (Table 4 ) show that the relative risk of having a Restricted Non-kin $(\exp \beta=1.11, p<0.001)$ or Multigenerational Household: Older Integrated networks (exp $\beta=1.15$, $p<0.001)$ increased as the number of years of age increased, while the risk of having a Middle-aged Friends network decreased with increasing age $(\exp \beta=0.91, p<0.001)$ relative to those in Multigenerational Household: Younger Family networks. Older women had approximately half the relative risk of men for having a Middle-aged Friends (exp $\beta=$ $0.57, p<0.05$ ) or Restricted Non-kin network ( $\exp \beta=0.46, p<0.01$ ) relative to Multigenerational Household: Younger Family networks.

Older people in Middle-aged Friends networks relative to Multigenerational Household: Younger Family networks (the reference group) had a 16.28 times higher likelihood of being never married $(\exp \beta=16.28, p<$ o.001) and a 4.82 times higher likelihood of being divorced or separated $(\exp \beta=4.82, p<0.001)$ relative to married. The Restricted Non-kin networks had an 8.92 times higher likelihood of being never married $(\exp \beta=8.92, p<0.01)$, three times higher likelihood of being widowed $(\exp \beta=3.00, p<0.001)$ and 6.35 times higher likelihood of being divorced or separated (exp $\beta=6.35, p<0.001)$ than married, relative to the Multigenerational Household: Younger Family networks. The results showed that older people with Multigenerational Household: Older Integrated networks were 0.47 times less likely to be widowed ( $\exp \beta=$ $0.47, p<0.01)$ rather than married, compared to the reference network. 
T А В LE 4. Multinomial logistic regression estimates of covariate effects on latent class membership: expressed as relative risks

\begin{tabular}{|c|c|c|c|}
\hline & MOI versus MYF & MAF versus MYF & RNK versus MYF \\
\hline & \multicolumn{3}{|c|}{ Adjusted odds ratio (95\% confidence interval) } \\
\hline Age & $1.15(1.10-1.19) * * *$ & $0.91(0.88-0.96)^{* * *}$ & $1.11(1.07-1.16)^{* * *}$ \\
\hline Gender (female) & $1.44(0.96-2.17)$ & $0.57(0.53-0.91) *$ & $0.46(0.30-0.72)^{* * *}$ \\
\hline \multicolumn{4}{|l|}{ Marital status: } \\
\hline Never married & $1.74(0.32-9.34)$ & $16.28(4.16-63.82)^{* * *}$ & $8.92(1.99-39.88) * *$ \\
\hline Widowed & $0.47(0.28-0.79) * *$ & $0.91\left(0.44^{-1.88)}\right.$ & $3.00(1.78-5.04)^{* * * *}$ \\
\hline $\begin{array}{l}\text { Divorced/ } \\
\text { separated }\end{array}$ & $0.73(0.31-1.72)$ & $4.82(2.54-9.15)^{* * *}$ & $6.35\left(3.23^{-12.49}\right) * * *$ \\
\hline Married & Ref. & Ref. & Ref. \\
\hline \multicolumn{4}{|l|}{ Ethnicity: } \\
\hline Chinese & $1.15\left(0.54^{-2.47}\right)$ & $0.97\left(0.44^{-2.11}\right)$ & $0.88\left(0.4^{2-1.85}\right)^{* * *}$ \\
\hline Black African & $0.7^{2}\left(0.3^{6}-1.4^{6}\right)$ & $0.31\left(0.15^{-0.65}\right)^{* *}$ & $0.35(0 / 18-0.69)^{* *}$ \\
\hline Indian & $0.97\left(0.49^{-1.93)}\right.$ & $0.53\left(0.25^{-1.12}\right)$ & $0.42(0.21-0.86)^{*}$ \\
\hline Pakistani & $0.74(0.37-1.45)$ & $0.29(0.13-0.64) * *$ & $0.30(0.14-0.61)^{* * *}$ \\
\hline Bangaladeshi & $0.59(0.29-1.20)$ & $0.20(0.08-0.49) * * *$ & $0.13(0.05-0.30) * * *$ \\
\hline Black Caribbean & Ref. & Ref. & Ref. \\
\hline \multicolumn{4}{|l|}{ Health status: } \\
\hline Good & $1.17(0.67-2.03)$ & $1.33\left(0.7^{0}-2.5^{1}\right)$ & $1.23(0.68-2.22)$ \\
\hline Fair & $1.91(1.15-3.17)^{*}$ & $1.55(0.80-2.98)$ & $2.48(1.43-4.32) * * *$ \\
\hline Poor & Ref. & Ref. & Ref. \\
\hline Pseudo $R^{2}$ & & 0.36 & \\
\hline
\end{tabular}

Notes: MOI: Multigenerational Household: Older Integrated. MYF: Multigenerational Household: Younger Family. MAF: Middle-aged Friends. RNK: Restricted Non-kin. Ref.: reference category.

Significance levels: $* p<0.05, * * p<0.01, * * * p<0.001$

For older people who believed that they were in 'fair health', the adjusted odds ratio (AOR) of being in Restricted Non-kin or a Multigenerational Household: Older Integrated network were approximately two and half $(\exp \beta=2.48, p<0.001)$ and two times greater $(\exp \beta=1.91, p<0.05)$ than having Multigenerational Household: Younger Family networks.

The relative risk of ethnic group influencing membership in a Multigenerational Household: Older Integrated network rather than a Multigenerational Household: Younger Family network was not significant. However, the AOR demonstrated that each ethnic group had a lower risk of being in a Restricted Non-kin network than a Multigenerational Household: Younger Family network (Chinese: $\exp \beta=0.88, p<0.001$; Black Africans: $\exp \beta=0.35, p<0.01$; Indian: $\exp \beta=0.42, p<0.05$; Pakistani: $\exp \beta=$ o.3o, $p<0.001$; Bangladeshi: $\exp \beta=0.13, p<0.001)$ when compared to Black Caribbeans. Furthermore, Black Africans $(\exp \beta=0.31, p<0.01)$, Pakistanis $(\exp \beta=0.29, p<0.01)$ and Bangladeshis $(\exp \beta=0.20, p<$ o.oo1) had a lower risk of having a Middle-aged Friends network than a Multigenerational Household: Younger Family network. 
After controlling for demographic variables, the analysis only partially supported our second hypothesis that the communalist cultures of Black Caribbean and Black African participants would manifest in a greater proportion of friends-based networks than other collectivist cultures. While Black Caribbean participants demonstrated a greater risk of having Middle-aged Friends networks compared to Multigenerational Household: Younger Family networks than Pakistani, Bangladeshi or Black African participants, there was no significant difference between this group and Indian and Chinese participants. Moreover, Black African participants had a higher risk of Multigenerational Household: Younger Family networks than Middle-aged Friends networks. Consequently, our third hypothesis that Chinese, Bangladeshi, Pakistani and Indian migrants will have a greater proportion of family-based networks than Black Caribbean and Black African migrants was also only partially supported. Only Bangladeshi and Pakistani participants had a greater risk of Multigenerational Household: Younger Family networks than Black Caribbean participants. Our fourth hypothesis that Chinese and Black Caribbean migrants would have a greater proportion of Restricted Non-kin networks than the other migrant groups was also only partially supported as Black Caribbean (but not Chinese) participants had a greater risk of being in a Restricted Non-kin network than a Multigenerational Household: Younger Family network.

\section{Outcomes of network membership}

In the first step of linear regression, demographic covariates were entered into the model with loneliness as the dependent variable. For the statistically significant predisposing variables, older people who were Chinese, female, had never married, or were divorced or separated, and in poor health experienced more loneliness than others (Table 5 ). The model explained 10.1 per cent of variance in loneliness. Adding variables representing the probability of classification to three networks explained an additional 1.5 per cent in variance: the combined model (Model 2) was statistically significant and explained 11.6 per cent of variance in loneliness. In Model 2, divorced and health status remained statistically significant but gender and 'never married' no longer significantly contributed to the model. Each network type was significantly associated with greater levels of loneliness (than those in Multigenerational Household: Younger Family networks). The analysis supports the fifth hypothesis that migrants with family-oriented networks will experience lower levels of loneliness.

With regard to the models explaining quality of life, the statistically significant predisposing variables demonstrated that older people that were divorced or separated, and in poor health experienced worse quality of 
T А B L 5 . Linear regression of loneliness and quality of life on personal characteristics and network type

\begin{tabular}{|c|c|c|c|c|c|c|c|c|}
\hline & \multicolumn{4}{|c|}{ Loneliness } & \multicolumn{4}{|c|}{ Quality of life } \\
\hline & \multicolumn{2}{|c|}{ Model 1} & \multicolumn{2}{|c|}{ Model 2} & \multicolumn{2}{|c|}{ Model 3} & \multicolumn{2}{|c|}{ Model 4} \\
\hline & $\beta$ & SE & $\beta$ & $\mathrm{SE}$ & $\beta$ & $\mathrm{SE}$ & $\beta$ & $\mathrm{SE}$ \\
\hline Age & -0.01 & 0.01 & -0.02 & 0.01 & -0.00 & 0.00 & -0.01 & 0.01 \\
\hline Gender (female) & $0.31 *$ & 0.15 & 0.29 & 0.15 & 0.07 & 0.05 & & \\
\hline \multicolumn{9}{|l|}{ Marital status: } \\
\hline Never married & $0.87^{*}$ & 0.44 & 0.69 & 0.45 & -0.04 & 0.14 & -0.11 & 0.15 \\
\hline Widowed & 0.26 & 0.19 & 0.27 & 0.20 & 0.04 & 0.06 & 0.03 & 0.06 \\
\hline Divorced/separated & $0.63 *$ & 0.25 & $0.5^{1 *}$ & 0.26 & $0.26 * * *$ & 0.08 & $0.20 *$ & 0.08 \\
\hline Married & Ref. & & Ref. & & Ref. & & Ref. & \\
\hline \multicolumn{9}{|l|}{ Ethnicity: } \\
\hline Chinese & $0.68 *$ & 0.28 & o. $68^{*}$ & 0.28 & 0.09 & 0.09 & 0.09 & 0.09 \\
\hline Black African & -0.04 & 0.25 & 0.07 & 0.25 & -0.08 & 0.08 & -0.03 & 0.08 \\
\hline Indian & $-0.4^{1}$ & 0.26 & $-0.3^{6}$ & 0.26 & $-0.26 * *$ & 0.08 & $-0.24^{* *}$ & 0.08 \\
\hline Pakistani & 0.23 & 0.26 & 0.34 & 0.26 & -0.11 & 0.08 & -0.07 & 0.08 \\
\hline Bangaladeshi & 0.17 & 0.27 & 0.34 & 0.27 & 0.11 & o.o8 & $0.18 *$ & 0.08 \\
\hline Black Caribbean & Ref. & & Ref. & & Ref. & & Ref. & \\
\hline \multicolumn{9}{|l|}{ Health status: } \\
\hline Good & $-1.37^{* * *}$ & 0.21 & $-1.3^{8 * * *}$ & 0.20 & $-1.22 * * *$ & 0.07 & -1.22 & 0.07 \\
\hline Fair & $-1.04 * * *$ & 0.19 & $-1.11 * * *$ & 0.19 & $-0.91 * * *$ & 0.06 & -0.94 & 0.06 \\
\hline Poor & Ref. & & Ref. & & Ref. & & Ref. & \\
\hline \multicolumn{9}{|l|}{ Network type: } \\
\hline MOI & & & $0.70^{* *}$ & 0.25 & & & $0.20^{*}$ & 0.08 \\
\hline MAF & & & $0.53^{*}$ & 0.27 & & & 0.15 & 0.09 \\
\hline RNK & & & $0.68 * *$ & 0.26 & & & $0.34^{* * *}$ & 0.08 \\
\hline MYF & & & Ref. & & & & Ref. & \\
\hline$R^{2}$ & 0.1 & & 0.1 & & 0.3 & & 0.3 & \\
\hline
\end{tabular}

Notes: SE: standard error. MOI: Multigenerational Household: Older Integrated. MAF: Middle-aged Friends. RNK: Restricted Non-kin. MYF: Multigenerational Household: Younger Family. Ref.: reference category.

Significance levels: $* p \leqslant 0.05, * * p \leqslant 0.01, * * * p \leqslant 0.001$. 
life than others (Table 5). Conversely, Indians had greater quality of life than other ethnic groups. The model explained 36.9 per cent of variance in quality of life. Adding the probability of classification to networks types explained an additional 1.3 per cent in variance and the combined model (Model 4 ) was statistically significant, accounting for 38.4 per cent of variance in quality of life. In Model 4 , being Indian remained statistically significant while being Bangladeshi became significantly associated with worse quality of life. Multigenerational Household: Older Integrated and Restricted Non-kin networks were associated with worse quality of life. Consequently, our sixth hypothesis that migrants with family-based networks would have a better quality of life than those with diverse or restricted networks was only partially supported. While migrants with Multigenerational Household: Younger Family networks had a greater quality of life than those with Multigenerational Household: Older Integrated and Restricted Non-kin networks, there was no significant difference in quality of life between people with these networks and those with Middle-aged Friends networks.

\section{Discussion}

The need for a culturally specific tool to identify older people who may need help from formal services is particularly pertinent in the UK, where research evidence with South Asian migrants suggests that not all extended families are willing or able to support older migrants (Katbamna et al. 2004). The UK has experienced a demographic shift towards greater proportions of ethnic minority elders (Burholt 2004a, 2004b). Following the Second World War, the UK experienced in-migration to fill labour shortages. Migrants tended to come from Commonwealth countries, particularly the Caribbean, Africa and the Indian sub-continent (including Pakistan and Bangladesh) (Burholt 2004a). As a consequence, until recently (and unlike the situation in the USA), there have been very few older people in these populations. This article has confirmed that the network typology that was developed with an older South Asian population (Burholt and Dobbs 2014) can be identified in other older populations with collectivist cultures. We have demonstrated that a four-profile model fits the data well, and that the profiles have characteristics that are very similar to the original development model.

Our analysis showed that the distribution of networks varied across ethnic groups. While we have considered a distinction between individualist and collectivist cultures, we have demonstrated that this is not a continuum and that there are different elements (communalism, familism, filial 


\section{Vanessa Burholt et al.}

piety, acculturation) that may impact on the configuration of support networks. This is particularly important when one considers the prevalence of the most and least robust networks. In this respect, while all network types were observed within each of the six ethnic groups, some had a greater proportion of robust networks than others. Of particular concern is the proportion $\left(3^{0.2 \%}\right)$ of older Black Caribbean migrants with Restricted Non-kin networks. Other research suggests that social exclusion may contribute to a lack of local social capital within this particular group, and that geographic dispersal of Black Caribbean populations coupled with inter-islander conflict (e.g. Jamaican versus Trinidadian) leads to divisions within this group (Campbell and McClean 2002).

Despite differences in the distribution of configuration of kin- and nonkin-based networks between ethnic groups, our results suggest that all of the migrant groups studied hold certain expectations concerning the role of the family. On the whole, the Multigenerational Household: Younger Family networks appear to be the desired network type in the collectivist cultures examined in this study. These networks are family focused networks and demonstrate normative differences in networks between collectivist and individualistic cultures. Locally integrated or diverse networks that have a high salience of contact with friends, family and involvement in community (and bear some similarities to the Multigenerational Household: Older Integrated or Middle-aged Friends networks) are more robust in individualistic cultures and less prone to loneliness and other negative wellbeing outcomes (Fiori, Antonucci and Cortina 20o6; Litwin and Shiovitz-Ezra 2010; Wenger 1991). This, however, is not the case in collectivist cultures. Contrary to individualistic cultures, we found that the most robust networks are privatised family-focused networks that include few non-kin members, that is those that we called Multigenerational Household: Younger Family networks. Deviation in network configuration resulted in worse wellbeing outcomes for older migrants, in terms of worse quality of life (with the exception of Middle-aged Friends) and greater loneliness. Thus, the cultural normative expectations about sources of support and family forms have a bearing on the extent to which networks can protect or buffer an older person from adverse outcomes.

Restricted Non-kin networks were most vulnerable: older people with these networks are more prone to 'fair' health, poor quality of life and loneliness than those with Multigenerational Household: Younger Family network types. However, the characteristics of Restricted Non-kin networks are again different to those observed in individualistic cultures. Whereas the latter tend to comprise only those living alone or with a spouse/partner that have few other ties, in collectivist cultures Restricted Non-kin networks may also include additional person $(s)$ (mean network size $=2.67$ ). Our choice of 
models was based on statistical fit to the data, but also theoretical reasoning, and in this respect we rejected the more strictly defined models in favour of larger networks. These networks are still small relative to other networks, and the fact that they are associated with negative wellbeing (in terms of quality of life and loneliness) suggests that upward social comparison to older people with larger family-based networks has a negative impact on the individual.

\section{Limitations}

We have identified two limitations to this study, and we address these in turn. Firstly, there were six distinct ethnic groups under investigation. Whilst we believe that the identification of the network types was contextually and statistically sound, it does not necessarily follow that our typology can be extrapolated to other ethnic populations and/or collectivist cultures (e.g. Litwin 2009). Secondly, the PAF sampling method ensures that where a higher density of a specific (ethnic) group is to be found, there will be a higher number of participants recruited. Given the importance of proximity to kin/friend in the typology classification process, it is possible that we have an under-representation of Restricted Non-kin networks. Therefore, we recommend that the network typology is further tested with indigenous and migrant populations with collectivist cultures and also in areas where migrant communities are more dispersed.

\section{Implications}

Despite the limitations noted above, we believe we have developed a useful model to examine network types within collectivist cultures. The typology is able to differentiate between networks with multigenerational households, and crucially it can help identify vulnerable networks. Consequently, the results of this research have important implications for forecasting formal services provision based on the distribution of support network types. Using network typologies that have not been developed for collectivist cultures may result in the amplification of the proportion of older people with robust networks (Burholt and Dobbs 2014). This, in turn, may contribute to tenacious stereotyping - that they prefer to 'look after their own' - and reinforce institutional racism: the belief of service providers that there is little that needs to be done in the way of service provision (Willis 2010). Service planning built on this evidence could underestimate the support needs of some older people who may be lonely, with poor quality of life and with limited informal sources of help. 


\section{Vanessa Burholt et al.}

The differences that are observed between the six ethnic groups in the $\mathrm{UK}$ indicate that more research is required on variation between cultures (Whitfield et al. 2008). For example, in the USA, differences between cultural sub-groups such as Mexican Americans, Latin Americans and Puerto Ricans may be lost when they are grouped together under one 'ethnic umbrella' such as 'Hispanics' (Whitbourne et al. 2005). In addition to impacting on planning of services, the use of this typology could impact on the credibility of gerontological research. Research that takes into account cultural variation is more likely to be better received by older people from different cultural groups, because it will be perceived as having more relevance to their lived experiences (Whitfield et al. 2008).

\section{Acknowledgements}

This work was supported by the Leverhulme Trust $(\mathrm{F} / \mathrm{oo275} / \mathrm{Q})$ and the National Institute of Social Care and Health Research (SCRA/10/02). We would like to thank Dr Wendy Martin, Dr Akile Ahmet, Dr Stefanie Doebler and Ethnic Focus for the contributions made to the research project. We acknowledge that the article could not be written without the contribution of the older people from ethnic groups in England and Wales who took the time to respond to our survey. Vanessa Burholt was the principal investigator of the study in Wales and Christina Victor was the principal investigator of the study in England. Both developed the ideas, study design and methods, and managed the research team collecting and analysing the data in the respective sites. Vanessa Burholt contributed to data analysis and drafted the manuscript. Christina Victor helped to prepare the manuscript. Christine Dobbs contributed to the data analysis and helped to draft the manuscript. All authors read and approved the final manuscript. Statement of conflict of interest: Professor Christina Victor is the Editor of the journal Ageing $\mathcal{E}$ Society.

\section{References}

Akaike, H. 1987. Factor analysis and AIC. Psychometrika, 52, 3, 317-32.

Burholt, V. 2004a. The settlement patterns and residential histories of older Gujaratis, Punjabis and Sylhetis in Birmingham, England. Ageing $\mathcal{E}^{\circ}$ Society, 24, $3,383-410$.

Burholt, V. 2004 $b$. Transnationalism, economic transfers and families' ties: intercontinental contacts of older Gujaratis, Punjabis and Sylhetis in Birmingham with families abroad. Ethnic and Racial Studies, 27, 5, 800-29.

Burholt, V. and Dobbs, C. 2014. A support network typology for application in older populations with a preponderance of multigenerational households. Ageing $\mathcal{E}^{\circ}$ Society, 34, 8, $1142-69$.

Burholt, V., Dobbs, C. and Victor, C. 2016. Transnational family relationships and cultural identity: older migrants in England and Wales. GeroPsych: The Journal of Gerontopsychology and Geriatric Psychiatry, 29, 2, 57-69. 
Burholt, V., Wenger, G. C., Scott, A., Yahya, B. and Roy, S. 20oo. Bangladeshi immigration to the United Kingdom: older people's support networks in the sending and receiving countries. Quality in Ageing, 1, 2, 18-30.

Burholt, V., Windle, G., Ferring, D., Balducci, C., Fagerström, C., Thissen, F., Weber, G. and Wenger, G. C. 2007. Reliability and validity of the Older Americans Resources and Services (Oars) Social Resources Scale in six European countries. Journals of Gerontology: Social Sciences, 62B, 6, S371-9.

Campbell, C. and McLean, C. 2002. Ethnic identity, social capital and health inequalities: factors shaping African-Caribbean participation in local community networks. Social Science $\mathcal{E}$ Medicine, 55, 4, 643-57.

Celeux, G. and Soromenho, G. 1996. An entropy criterion for assessing the number of clusters in a mixture model. Journal of Classification, 13, 2, 195-212.

Cheng, S.-T., Fung, H. and Chan, A. 2007. Maintaining self-rated health through social comparison in old age. Journals of Gerontology: Psychological Sciences, 62 B, 5 , $\mathrm{P}_{277}-85$.

Cheng, S.-T., Lee, C. K. L., Chan, A. C. M., Leung, E. M. F. and Lee, J-J. 2oog. Social network types and subjective well-being in Chinese older adults. Journals of Gerontology: Psychological Sciences, 64B, 6, 71 3-22.

De Jong Gierveld, J. and Van Tilburg, T. 2006. A 6-item scale for overall, emotional and social loneliness. Research on Ageing, 25, 5, 582-98.

Dubova, S. V., Pérez-Cuevas, R., Espinosa-Alarcón, P. and Flores-Hernández, S. 2010. Social network types and functional dependency in older adults in Mexico. BMC Public Health, 10, 104.

Festinger, L. 1954. A theory of social comparison processes. Human Relations, 7, 2, $117-40$.

Fiori, K. L., Antonucci, T. C. and Cortina, K. S. 20o6. Social network typologies and mental health among older adults. Journals of Gerontology: Psychological Sciences and Social Sciences, 61B, 1, $\mathrm{P}_{25} 5^{-32 .}$

Golden, J., Conroy, R. M. and Lawlor, B. A. 2009. Social support network structure in older people: underlying dimensions and association with psychological and physical health. Psychology, Health $\mathcal{E}$ Medicine, 14, 3, 280-9o.

Grant, R. L. and Bowling, A. 2011 . Challenges in comparing the quality of life of older people between ethnic groups, and the implications for national wellbeing indicators: a secondary analysis of two cross-sectional surveys. Health and Quality of Life Outcomes, 9, 1, 109.

Henry, D. B., Tolan, P. H. and Gorman-Smith, D. 2005. Cluster analysis in family psychology research. Journal of Family Psychology, 19, 1, $121-32$.

Hofstede, G. 2001. Culture's Consequences: Comparing Values, Behaviors, Institutions, and Organizations Across Nations. Sage, Thousand Oaks, California.

Katbamna, S., Ahmad, W., Bhakta, P., Baker, R. and Parker, G. 2004. Do they look after their own? Informal support for South Asian carers. Health $\mathcal{E}$ Social Care in the Community, 12, 5, 398-406.

Koller, M., Aaronson, N. K., Blazeby, J., Bottomley, A., Dewolf, L., Fayers, P., Johnson, C., Ramage, J., Scott, N., West, K. and the EORTC Quality of Life Group 2007. Translation procedures for standardised quality of life questionnaires: the European Organisation for Research and Treatment of Cancer (EORTC) approach. European Journal of Cancer, 43, 12, 1810-20.

Knight, B. G. and Sayegh, P. 20og. Cultural values and caregiving: the updated sociocultural stress and coping model. Journals of Gerontology: Psychological Sciences $\mathcal{E}$ Social Sciences, $\mathbf{6}_{\mathbf{5}} \mathbf{B}, 1,5^{-1} 3$.

Litwin, H. 1997. Support network type and health services utilization. Research on Aging, 19, 3, 274-99. 


\section{Vanessa Burholt et al.}

Litwin, H. 2009. Social networks and well-being: a comparison of older people in Mediterranean and non-Mediterranean countries. Journals of Gerontology: Social Sciences, 65 $\mathbf{B}, 5,599-608$.

Litwin, H. and Shiovitz-Ezra, S. 2010. Social network type and subjective well-being in a national sample of older Americans. The Gerontologist, 51, 3, 379-88.

Lubben, J. and Gironda, M. 2003. Centrality of social ties to the health and wellbeing of older adults. In Berkman, B. and Harooytan, L. K. (eds), Social Work and Health Care in an Aging World. Springer, New York, 319-50.

Lubben, J. and Gironda, M. 2004. Measuring social networks and assessing their benefits. In Phillipson, C., Allan, G. and Morgan, D. (eds), Social Networks and Social Exclusion: Sociological and Policy Perspective. Ashgate, Aldershot, UK, 20-34.

McLachlan, G. and Peel, D. 20oo. Finite Mixture Models. Wiley, New York.

National Health Service Information Centre 2010. Comparison of Results from Surveys of Carers, 2009-10. National Health Service Information Centre, Leeds, UK.

Nylund, K. L. 2007. Deciding on the number of classes in latent class analysis and growth mixture modeling: a Monte Carlo simulation study. Structural Equation Modeling, 14, 4, 535-69.

Royal Mail 2009. Technical Specifications for Users of PAF $F^{\circledR}$ and Associated Raw Data Products: Programmers' Guide. Edition 7, Version 5.o, Royal Mail Group, Portsmouth, UK.

Schwartz, S. J., Weisskirch, R. S., Hurley, E. A., Zamboanga, B. L., Park, I. J. K., Kim, S. Y., Umaña-Taylor, A., Castillo, L. G., Brown, E. and Greene, A. D. 2010. Communalism, familism and filial piety: are they birds of a collectivist feather? Cultural Diversity E Ethnic Minority Psychology, 16, 4, 548-6o.

Sclove, L. 1987 . Application of model-selection criteria to some problems in multivariate analysis. Psychometrika, 52, 3, 333-43.

Triandis, H. C. 1989. The self and social behavior in differing cultural contexts. Psychological Review, 96, 3, 506-20.

Triandis, H.C. 1995. Individualism and Collectivism. Westview Press, Boulder, Colorado.

Triandis, H. C., Bontempo, R., Villareal, M.J., Asai, M. and Lucca, N. 1988. Individualism and collectivism: cross-cultural perspectives on self-ingroup relationships. Journal of Personality and Social Psychology, 54, 2, 323-38.

van de Vijver, F.J. R. and Arends-Tóth, J. V. 2009. Family support in five cultural groups in the Netherlands. Journal of Comparative Family Studies, 4o, 3, 455-73.

Vuong, Q. 1989. Likelihood ratio tests for model selection and non-nested hypotheses. Econometrica, 57, 2, 307-33.

Wallace, B. C. and Constantine, M. G. 2005. Africentric cultural values, psychological help-seeking attitudes, and self-concealment in African American college students. Journal of Black Psychology, 31, 4, 369-85.

Walthery, P., Stafford, M., Nazroo, J., Whitehead, M., Dibben, C., Halliday, E., Povall, S. and Popay, J. 2015. Health trajectories in regeneration areas in England: the impact of the New Deal for Communities intervention. Journal of Epidemiology E Community Health, 69, 8, 762-68.

Wenger, G. C. 1991. A network typology: from theory to practice. Journal of Aging Studies, 5, 2, 147-62.

Wenger, G. C., Davies, R., Shahtahmasebi, S. and Scott, A. 1996. Social isolation and loneliness in old age: review and model refinement. Ageing $\mathcal{E}^{2}$ Society, 16, 3, $333^{-} 5^{8}$.

Wenger, G. C. and Liu, J. M. 1999. Support networks in Beijing (China) and Liverpool (UK): differences and similarity. Hallym International Journal of Aging, 1, 2, 47-57. 
Whitbourne, S. K., Bringle, J. R., Yee, B., Chiriboga, D. and Whitfield, K. 2005. Community research settings for the elderly. In Trimble, J. E. and Fisher, C. B. (eds), Handbook of Ethical Research with Ethnocultural Populations and Communities. Sage, Thousand Oaks, California, $217-40$.

Whitfield, K. E., Allaire, J. C., Belue, R. and Edwards, C. L. 2008. Are comparisons the answer to understanding behavioral aspects of aging in racial and ethnic groups? Journals of Gerontology: Psychological Sciences, $\mathbf{6 3}_{3} \mathbf{B}, 5, \mathrm{P}_{301}-8$.

Willis, R. 2008. Advantageous inequality or disadvantageous equality? Ethnicity and family support among older people in Britain. Ethnicity and Inequalities in Health and Social Care, 1, 2, 18-23.

Willis, R. 2010. Myths and statistics: an exploration of the assumptions connected to ethnicity and family support. Radical Statistics, 10o, $17-27$.

Willis, R. 2012 . Individualism, collectivism and ethnic identity: cultural assumptions in accounting for caregiving behaviour in Britain. Journal of Cross-cultural Gerontology, 27, 3, 201-16.

Accepted 5 January 2017 ; first published online 27 February 2017

Address for correspondence:

Vanessa Burholt, Room 2o Haldane Building,

Centre for Innovative Ageing, College of Human and Health Sciences,

Swansea University, Singleton Park,

Swansea SA2 8PP, UK

E-mail: v.burholt@swansea.ac.uk 